\title{
38. REFLECTION NEBULAE AND SPIRAL STRUCTURE
}

\author{
R. RACINE and S. VAN DEN BERGH \\ David Dunlap Observatory, Richmond Hill, Ontario, Canada
}

\begin{abstract}
It is shown that stars embedded in reflection nebulosity may be used as spiral-arm tracers.
Surveys of stars embedded in reflection nebulae (Dorschner and Gürtler, 1964, 1965; Van den Bergh, 1966) show that reflection nebulae occur in associations. A detailed photometric and spectroscopic investigation of these associations of reflection nebulae has been carried out by Racine $(1968,1970)$. Some $R$ associations coincide with known OB associations, others are connected with groupings of $\mathrm{T}$ Tauri stars and some are located in regions in which star formation was not previously known to occur.

Racine (1968) and Van den Bergh (1968) were able to show that $\mathrm{R}$ associations outline the Orion spiral arm rather better than do OB associations. The reason for this is that the number of $\mathrm{R}$ associations per $\mathrm{kpc}^{2}$ in the galactic plane is greater than is the number of OB associations. This is so because the late B and early A stars, that dominate the stellar content of $\mathrm{R}$ associations, have a much greater space density than do the $\mathrm{O}$ and early $\mathrm{B}$ stars which populate $\mathrm{OB}$ associations.
\end{abstract}

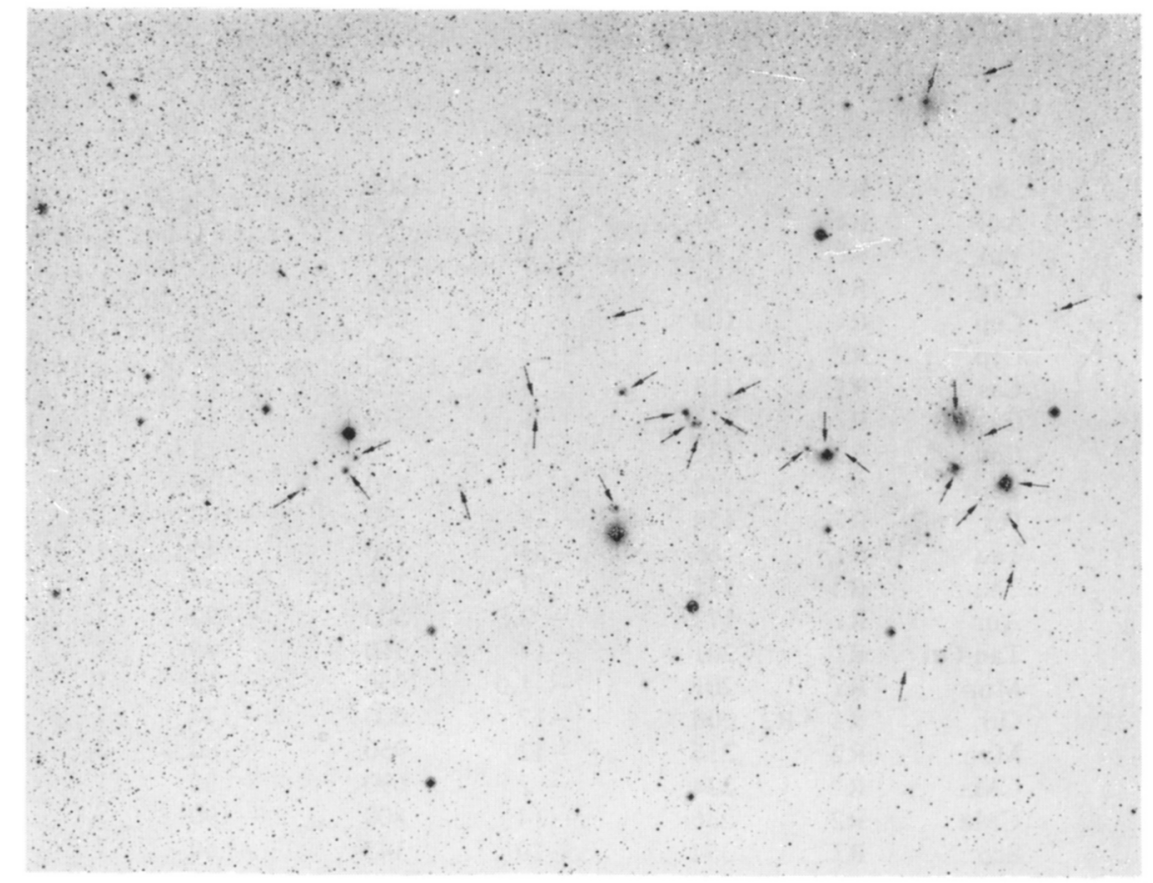

Fig. 1. Mon $\mathbf{R} 2$ is a good example of an association of reflection nebulae. The earliest spectral type in this $\mathrm{R}$ association is $\mathrm{B} 1$. The distance of the association Mon R 2 is $950 \mathrm{pc}$. 
A good example of an association of reflection nebulae is shown in Figure 1. In the figure 27 members of the association Mon R 2 are identified. This association, which contains both early and late B stars, is located at a distance of $950 \mathrm{pc}$.

Table I lists the associations of reflection nebulae that have so far been studied in detail. The positions of these $\mathrm{R}$ associations are plotted in Figure 2. The figure shows that associations of reflections nebulae outline the Orion spiral arm rather well. Figure 2 shows that this arm extends from $l^{\mathrm{II}}=70^{\circ}$ to $l^{\mathrm{II}}=250^{\circ}$. In the direction $l^{\mathrm{II}}=160^{\circ}$ (Perseus-Auriga) the principal axis of the Orion arm, as outlined by reflection nebulae, is located at a distance of approximately $250 \mathrm{pc}$ from the sun. Comparison of Figure 2 with plots of the distribution of clusters, OB associations and HII regions (Becker, 1964) shows excellent agreement between the spiral arms outlined by $\mathrm{R}$ associations and those obtained by other methods. The apparent lack of $\mathrm{R}$ associations in the Southern Milky Way is entirely due to observational selection. The Curtis Schmidt telescope on Cerro Tololo will shortly be used for a systematic survey of southern reflection nebulae.

One of the most striking features revealed by the distribution of $\mathrm{R}$ associations is that the Orion spiral arm is inclined to the galactic plane. In Cygnus and Cepheus the Orion arm is located approximately $50 \mathrm{pc}$ above the plane; the Orion arm crosses the galactic planc in Cassiopeia and remains below the plane from Perseus to Canis Major.

TABLE I

Data on associations of reflection nebulae

\begin{tabular}{|c|c|c|c|c|c|c|}
\hline \multicolumn{2}{|c|}{ Association } & \multirow{2}{*}{$\begin{array}{l}l^{\mathrm{II}} \\
13\end{array}$} & \multirow{2}{*}{$\begin{array}{l}b^{\text {II }} \\
-0.8\end{array}$} & \multirow{2}{*}{$\begin{array}{l}d(\mathrm{pc}) \\
1200\end{array}$} & \multirow{2}{*}{$\begin{array}{l}z(\mathrm{pc}) \\
-\quad 17\end{array}$} & \multirow{2}{*}{$\begin{array}{l}\text { References } \\
1\end{array}$} \\
\hline Sgr & R1 & & & & & \\
\hline Aql & R1 & 30 & +4 & 380 & +27 & 4 \\
\hline Vul & R1 & 67 & -1.1 & 2500 & -40 & 4 \\
\hline Cyg & R1 & 77 & +1.5 & 1000 & +26 & 1 \\
\hline Cep & $\mathbf{R} 1$ & 109 & +4 & 650 & +46 & 1 \\
\hline Cep & $\mathbf{R} 2$ & 111 & +12 & 400 & +83 & 1 \\
\hline Cas & R1 & 118 & -3 & 520 & -27 & 1 \\
\hline Cas & $\mathbf{R} 2$ & 133 & +8 & 310 & +43 & 4 \\
\hline Cas & R3 & 131 & +1 & 750 & +13 & 4 \\
\hline Cam & R1 & 142 & +2 & 870 & +30 & 1 \\
\hline Per & R1 & 158 & -18 & 330 & -100 & 1 \\
\hline Tau & R1 & 166 & -24 & 110 & -45 & 1 \\
\hline Tau & $\mathbf{R} 2$ & 171 & -17 & 135 & -39 & 1 \\
\hline Aur & $\mathrm{R} 1$ & 173 & -3.5 & 1500 & -90 & 4 \\
\hline Tau-Ori & R1 & 201 & -17 & 360 & -105 & 1 \\
\hline Mon & $\mathbf{R} 1$ & 201 & -1.0 & 1050 & -18 & 1 \\
\hline Ori & $\mathbf{R} 1+\mathbf{R} 2$ & 208 & -17 & 600 & -170 & 1 \\
\hline Mon & $\mathbf{R} 2$ & 216 & -12 & 950 & -180 & 2 \\
\hline $\mathrm{CMa}$ & R1 & 224 & -2 & 690 & -30 & 1 \\
\hline CMa & $\mathrm{R} 2$ & 236 & -14 & 800 & -190 & 3 \\
\hline Sco & $\mathbf{R} 1$ & 354 & +20 & 145 & +50 & 1 \\
\hline
\end{tabular}

$1=$ Racine (1968); $2=$ Racine (1968) revised; $3 \cdots$ Van den Bergh (1968); 4 Racine (1970). 


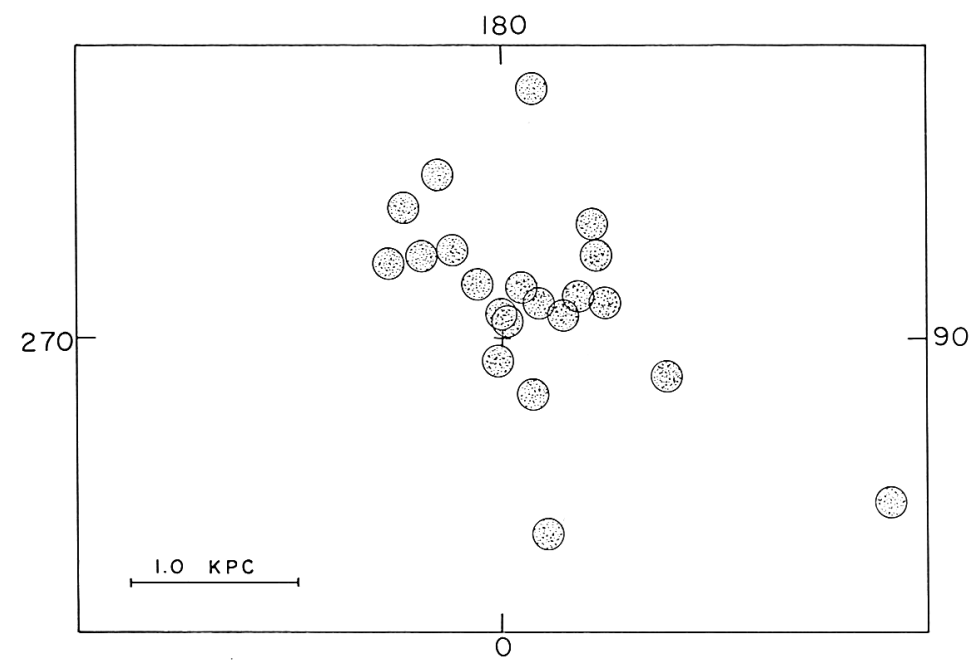

Fig. 2. Plot of the positions of $\mathbf{R}$ associations projected on the galactic plane. The position of the sun is marked by a cross.

The most southern $\mathrm{R}$ association that has so far been observed in the Orion Spiral arm is CMa R2 (Van den Bergh, 1968) which is situated $190 \mathrm{pc}$ below the galactic plane. Additional southern hemisphere observations will be required to see if the Orion arm peters out at this point or whether it continues on and dips down again into the galactic plane.

\section{References}

Becker, W.: 1964, Z. Astrophys. 58, 202.

Dorschner, J. and Gürtler, J.: 1964, Astron. Nachr. 287, 257.

Dorschner, J. and Gürtler, J.: 1965, Astron. Nachr. 289, 57.

Racine, R.: 1968, Astron. J. 73, 233.

Racine, R.: 1970, in preparation.

Van den Bergh, S.: 1966, Astron. J. 71, 990.

Van den Bergh, S.: 1968, Astrophys. Letters $2,71$. 